\title{
RADIATION AND CHEMICAL REACTION EFFECTS ON UNSTEADY MHD FREE CONVECTION PARABOLIC FLOW PAST AN INFINITE ISOTHERMAL VERTICAL PLATE WITH VISCOUS DISSIPATION
}

\author{
B. PRABHAKAR REDDY \\ Department of Mathematics \\ College of Natural and Mathematical Sciences \\ The University of Dodoma \\ P. Box. No: 338, Dodoma, TANZANIA \\ Geethanjali College of Engineering and Technology \\ Cheeryal (V), Keesara (M), Medchal (Dist) - 501301 \\ Telangana, INDIA \\ E-mail: prabhakar.bijjula@gmail.com
}

\begin{abstract}
The numerical investigation of the effects of radiation and chemical reaction on an unsteady MHD free convection flow with a parabolic starting motion of an infinite isothermal vertical porous plate taking into account the viscous dissipation effect has been carried out. The fluid is considered a gray, absorbing emitting radiation but a non-scattering medium. The dimensionless governing equations for this investigation are solved numerically by applying the Ritz finite element method. Numerical results for the velocity profiles, temperature profiles and concentration profiles as well as the skin-friction are presented through graphs and tables for different values of the physical parameters involved. Results obtained show a decrease in the temperature and velocity in the boundary layer as the radiation parameter increased. The velocity increases with an increase in the thermal and mass Grashof numbers and decreases with an increase in the magnetic parameter. Further, the concentration and velocity decreases with increasing the Schmidt number and chemical reaction parameter. These findings are in very good agreement with the studies reported earlier.
\end{abstract}

Key words: radiation parameter, chemical reaction parameter, porous medium, MHD, free convection, isothermal vertical plate.

\section{Introduction}

The study of magneto-hydrodynamics (MHD) with heat and mass transfer in the presence of radiation has attracted the attention of many researchers, due to diverse applications. In astrophysics and geophysics, it is functional to study the stellar structures, radio propagation through the ionosphere, etc. Radiative flow are encountered in many industrial and environmental processes, e.g., heating and cooling chambers, fossil fuel combustion energy processes, evaporation from large open water reservoirs, astrophysical flows, solar power technology and space vehicle re-entry etc. Muthucumaraswamy and Janakiraman [1] studied MHD and radiation effects on a moving isothermal vertical plate with variable mass diffusion. Shanker and Gnaneshwar [2] analyzed the effects of radiation on an MHD flow past an impulsively started infinite vertical plate through a porous medium with variable temperature and mass diffusion. Thermal radiation effects on a transient MHD flow with mass transfer past an impulsively fixed infinite vertical plate was investigated by Ahmed and Sarmah [5]. The effects of thermal radiation on an unsteady MHD free convection flow past a vertical plate with temperature dependent viscosity was investigated by Mahmoud [6]. Mukhopadhyay [7] presented the effects of thermal radiation on an unsteady mixed convection flow and heat transfer over a porous stretching surface in a porous medium. Sharma and Deka [8] analyzed thermal radiation and oscillating plate temperature effects on an unsteady MHD flow past 
a semi-infinite vertical porous plate in the presence of chemical reaction. The effect of radiation on a linearly accelerated plate with variable mass diffusion in the presence of the magnetic field was investigated by Muralidharan and Muthucumaraswamy [9].

The growing need for chemical reactions in chemical and hydrometallurgical industries requires the study of heat and mass transfer in the presence of chemical reaction. There are many transport processes that are governed by the combined action of buoyancy forces due to both thermal and mass diffusion in the presence of chemical reaction. These processes are observed in nuclear reactor safety and combustion systems, solar collectors as well as metallurgical and chemical engineering. Their other applications include solidification of binary alloys and crystal growth dispersions of dissolved materials or particular water in flows, drying and dehydration operations in chemical and food processing plants, and combustion of atomized liquid fuels. Muthucumaraswamy and Ganesan [10] analyzed the effect of chemical reaction and injection on flow characteristics in an unsteady upward motion of an isothermal plate. MHD flow of a uniformly stretched vertical permeable surface in the presence of heat generation /absorption and a chemical reaction was presented by Chamkha [11]. The effects of chemical reaction, heat and mass transfer along a wedge with heat source and concentration in the presence of suction or injection was investigated by Kandasamy et al. [12]. Ibrahim et al. [13] analyzed the effects of chemical reaction and radiation/absorption on the unsteady MHD free convection flow past a semi-infinite vertical permeable moving plate with heat source and suction. Muthucumaraswamy and Janakiraman [14] investigated mass transfer effects on an isothermal vertical oscillating plate in the presence of chemical reaction. Mahapatra et al. [15] presented the effects of chemical reaction on free convection flow through a porous medium bounded by a vertical surface. Unsteady MHD double-diffusive convection boundary layer flow past a radiative hot vertical surface in a porous media in the presence of chemical reaction and heat sink was studied by Mohamed et al. [16]. MHD free convection parabolic flow past an infinite isothermal vertical plate in the presence of thermal radiation and chemical reaction was investigated by Muthucumaraswamy and Sivakaumar [17].

The dissipation of energy is significant when considering unsteady magneto-hydrodynamic natural convection flows. The viscous dissipation effect is expected to be relevant for fluids with high values of dynamic viscosity as for high velocity flows. The viscous dissipation heat is important in the natural convective flows, when the field is of extreme size or at extremely low temperature or in high gravitational field. Gebhart [19] showed the importance of viscous dissipative heat in free convection flow in the case of an isothermal vertical plate with constant heat flux. Gebhart and Mollendorf [20] considered the effects of viscous dissipation for external natural convection flow over a surface. The viscous dissipation heat on the two-dimensional unsteady free convective flow past an infinite vertical porous plate when the temperature oscillates in time and there is constant suction at the plate was reported by Soundalgekar [21]. Gupta et al. [22] presented free convection effects on flow past a linearly accelerated vertical plate in the presence of viscous dissipative heat using the perturbation method. The computational analysis of coupled radiationconvection dissipation non-gray gas flow in a non-Darcy porous medium with the Keller-Box implicit difference scheme was reported by Takhar [23]. Zueco [24] investigated radiation and viscous dissipation effects on an unsteady MHD free convection flow over a vertical porous plate by the network simulation method. Recently, Reddy [25] studied mass transfer effects on an unsteady MHD free convective flow of an incompressible viscous dissipative fluid past an infinite vertical porous plate by the finite element method.

The object of the present work is to analyze the effects of radiation and chemical reaction on an unsteady MHD free convection with a parabolic starting motion of an infinite isothermal vertical porous plate taking into account the viscous dissipation effect. The problem is governed by the system of coupled non-linear partial differential equations, whose exact solutions are difficult to obtain, if possible. So, the Ritz finite element method has been adopted for its solution, which is more economical from the computational point of view. The purpose of this paper is to extend the results of Muthucumaraswamy and Sivakumar [17] especially with the incorporation of the viscous dissipation effect and the permeability of the porous medium. The influence of material parameters encountered in the problem under the investigation on the flow has been presented through graphs and tables and then discussed. 


\section{Mathematical analysis}

We consider an unsteady MHD flow of a viscous incompressible fluid with a parabolic started motion of an infinite isothermal vertical porous plate in the presence of radiation and chemical reaction of first order taking into account the viscous dissipation effect. The fluid is considered a gray, absorbing emitting radiation but a non-scattering medium. The $x^{\prime}$-axis is taken along the plate in the upward direction and the $y^{\prime}$-axis is taken normal to the plate. A magnetic field of strength $B_{0}$ is applied transversely to the direction of the flow. At time $t^{\prime} \leq 0$, the plate and fluid are at same temperature $T_{\infty}{ }^{\prime}$ and concentration level $C_{\infty}{ }^{\prime}$ at all points. At time $t^{\prime}>0$, the plate started with velocity $u^{\prime}=u_{0} t^{\prime 2}$ in its own plane against the gravitational field and the temperature from the plate rises to $T_{w}^{\prime}$ and the concentration level near the plate rises to $C_{w}^{\prime}$. A chemically reactive species which transforms according to a simple reaction involving the concentration is emitted from the plate and diffuses into the fluid. The reaction is assumed to take place entirely in the stream. Under the usual Boussinesq's approximation and boundary layer approximations, the unsteady parabolic starting motion is governed by the following equations:

$$
\begin{aligned}
& \frac{\partial u^{\prime}}{\partial t^{\prime}}=g \beta\left(T^{\prime}-T_{\infty}^{\prime}\right)+g \beta^{*}\left(C^{\prime}-C_{\infty}^{\prime}\right)+v \frac{\partial^{2} u^{\prime}}{\partial y^{\prime 2}}-\frac{v u^{\prime}}{K^{\prime}}-\frac{\sigma B_{0}^{2}}{\rho} u^{\prime}, \\
& \rho C_{p} \frac{\partial T^{\prime}}{\partial t^{\prime}}=k \frac{\partial^{2} T^{\prime}}{\partial y^{\prime 2}}-\frac{\partial q_{r}}{\partial y^{\prime}}+\mu\left(\frac{\partial u^{\prime}}{\partial y^{\prime}}\right)^{2}, \\
& \frac{\partial C^{\prime}}{\partial t^{\prime}}=D \frac{\partial^{2} C^{\prime}}{\partial y^{\prime 2}}-k_{l}\left(C^{\prime}-C_{\infty}^{\prime}\right)
\end{aligned}
$$

The boundary conditions for the velocity, temperature and concentration fields are

$$
\begin{aligned}
& t^{\prime} \leq 0 ; \quad u^{\prime}=0, \quad T^{\prime}=T_{\infty}^{\prime}, \quad C^{\prime}=C_{\infty}^{\prime} \quad \text { for all } \quad y^{\prime}=0, \\
& t^{\prime}>0 ; \quad u^{\prime}=u_{0} t^{\prime 2}, \quad T^{\prime}=T_{\infty}^{\prime}, \quad C^{\prime}=C_{\infty}^{\prime} \quad \text { at } \quad y^{\prime}=0, \\
& u^{\prime} \rightarrow 0, \quad T^{\prime} \rightarrow T_{\infty}^{\prime}, \quad C^{\prime} \rightarrow C_{\infty}^{\prime} \quad \text { at } \quad y^{\prime} \rightarrow \infty .
\end{aligned}
$$

The local radiant for the case of an optically thin gray gas is expressed as

$$
\frac{\partial q_{r}}{\partial y^{\prime}}=-4 a^{*} \sigma\left(T_{\infty}^{\prime}-T^{\prime^{4}}\right)
$$

It is assumed that the temperature differences within the flow are sufficiently small such that $T^{14}$ can be expressed as a linear function of the temperature. This is accomplished by expanding $T^{14}$ in a Taylor series about $T_{\infty}^{\prime}$ and neglecting the higher order terms. One obtains

$$
T^{\prime 4} \cong 4 T_{\infty}^{\prime} T^{\prime}-3 T_{\infty}^{\prime 4}
$$


Using Eqs (2.5) and (2.6), Eq.(2.2) reduces to

$$
\rho C_{p} \frac{\partial T^{\prime}}{\partial t^{\prime}}=k \frac{\partial^{2} T^{\prime}}{\partial y^{\prime 2}}-16 a^{*} \sigma T_{\infty}^{\prime 3}\left(T^{\prime}-T_{\infty}^{\prime}\right)+\mu\left(\frac{\partial u^{\prime}}{\partial y^{\prime}}\right)^{2} .
$$

Let us introduce the following non-dimensional quantities

$$
\begin{aligned}
& u=u^{\prime}\left(\frac{u_{0}}{v^{2}}\right)^{\frac{1}{3}}, \quad y=y^{\prime}\left(\frac{u_{0}}{v^{2}}\right)^{\frac{1}{3}}, t=t^{\prime}\left(\frac{u_{0}}{v^{2}}\right)^{\frac{1}{3}}, \operatorname{Pr}=\frac{\mu c_{p}}{k}, \quad \mathrm{Sc}=\frac{v}{D}, M=\frac{\sigma B_{0}^{2}}{\rho}\left(\frac{v}{u_{0}^{2}}\right)^{\frac{1}{3}}, \\
& K=K^{\prime}\left(\frac{u_{0}}{v^{2}}\right)^{\frac{2}{3}}, \theta=\frac{\left(T^{\prime}-T_{\infty}^{\prime}\right)}{\left(T_{w}^{\prime}-T_{\infty}^{\prime}\right)}, C=\frac{\left(C^{\prime}-C_{\infty}^{\prime}\right)}{\left(C_{w}^{\prime}-C_{\infty}^{\prime}\right)}, R=\frac{16 a^{*} \sigma T_{\infty}^{3}}{k}\left(\frac{v^{2}}{u_{0}}\right)^{\frac{2}{3}}, \delta=k_{l}\left(\frac{v}{u_{o}}\right)^{\frac{1}{3}}, \\
& H=M+\frac{1}{K}, \quad \mathrm{Ec}=\frac{v}{C_{p}\left(T_{w}^{\prime}-T_{\infty}^{\prime}\right)}, \quad \mathrm{Gr}=\frac{g \beta\left(T_{w}^{\prime}-T_{\infty}^{\prime}\right)}{\left(v u_{0}\right)^{1 / 3}}, G_{m}=\frac{g \beta^{*}\left(C_{w}^{\prime}-C_{\infty}^{\prime}\right)}{\left(v u_{0}\right)^{1 / 3}} .
\end{aligned}
$$

The governing system of Eqs (2.1), (2.3), (2.4) and (2.7) with the use of Eq.(2.8) is reduced to the following non-dimensional form

$$
\begin{aligned}
& \frac{\partial u}{\partial t}=\operatorname{Gr} \theta+G_{m} C+\frac{\partial^{2} u}{\partial y^{2}}-H u, \\
& \frac{\partial \theta}{\partial t}=\frac{1}{\operatorname{Pr}} \frac{\partial^{2} \theta}{\partial y^{2}}-\frac{R}{\operatorname{Pr}} \theta+\operatorname{Ec}\left(\frac{\partial u}{\partial y}\right)^{2}, \\
& \frac{\partial C}{\partial t}=\frac{1}{\operatorname{Sc}} \frac{\partial^{2} C}{\partial y^{2}}-\delta C
\end{aligned}
$$

The corresponding initial and boundary conditions in a non-dimensional form are

$$
\begin{aligned}
& t \leq 0 ; \quad u=0, \quad \theta=0, \quad C=0 \quad \text { for all } \quad y, \\
& t>0 ; \quad u=t^{2}, \quad \theta=1, \quad C=1 \quad \text { at } \quad y=0, \\
& u \rightarrow 0, \quad \theta \rightarrow 0, \quad C \rightarrow 0 \quad \text { as } \quad y \rightarrow \infty .
\end{aligned}
$$




\section{Method of solution}

The dimensionless governing Eqs (2.9) (2.11) are to be solved subject to the initial and boundary conditions given in Eqs (2.12) by applying the Ritz finite element method. The Ritz finite element method applied to Eq.(2.9) over the two-nodded linear element $(e),\left(y_{j} \leq y \leq y_{k}\right)$, yields

$$
J^{(e)}(u)=\frac{1}{2} \int_{y_{j}}^{y_{k}}\left\{\left(\frac{\partial u^{(e)}}{\partial y}\right)^{2}+H u^{(e)^{2}}+2 u^{(e)} \frac{\partial u^{(e)}}{\partial t}-2 u^{(e)} \Delta\right\} d y=\text { minimum }
$$

where

$$
\Delta=(\operatorname{Gr} \theta+\operatorname{Gm} C)
$$

Let $u^{(e)}=\psi_{j}(y) u_{j}(t)+\psi_{k}(y) u_{k}(t)=\psi_{j} u_{j}+\psi_{k} u_{k}$ be the linear approximation solution over the element $(e),\left(y_{j} \leq y \leq y_{k}\right)$. The element equation is given by

$$
\begin{aligned}
& \int_{y_{j}}^{y_{k}}\left[\begin{array}{rr}
\psi_{j}^{\prime} \psi_{j}^{\prime} & \psi_{j}^{\prime} \psi_{k}^{\prime} \\
\psi_{k}^{\prime} \psi_{j}^{\prime} & \psi_{k}^{\prime} \psi_{k}^{\prime}
\end{array}\right]\left[\begin{array}{l}
u_{j} \\
u_{k}
\end{array}\right] d y+H \int_{y_{j}}^{y_{k}}\left[\begin{array}{ll}
\psi_{j} \psi_{j} & \psi_{j} \psi_{k} \\
\psi_{k} \psi_{j} & \psi_{k} \psi_{k}
\end{array}\right]\left[\begin{array}{l}
u_{j} \\
u_{k}
\end{array}\right] d y+ \\
& +\int_{y_{j}}^{y_{k}}\left[\begin{array}{ll}
\psi_{j} \psi_{j} & \psi_{j} \psi_{k} \\
\psi_{k} \psi_{j} & \psi_{k} \psi_{k}
\end{array}\right]\left[\begin{array}{l}
u_{j}^{\cdot} \\
u_{k}^{\cdot}
\end{array}\right] d y-\Delta \int_{y_{j}}^{y_{k}}\left[\begin{array}{l}
\psi_{j} \\
\psi_{k}
\end{array}\right] d y=0
\end{aligned}
$$

where the prime and the dot denote differentiation with respect to $y$ and $t$, respectively. Simplifying yields

$$
\frac{1}{l^{(e)}}\left[\begin{array}{cc}
1 & -1 \\
-1 & 1
\end{array}\right]\left[\begin{array}{l}
u_{j} \\
u_{k}
\end{array}\right]+\frac{H l^{(e)}}{6}\left[\begin{array}{ll}
2 & 1 \\
1 & 2
\end{array}\right]\left[\begin{array}{l}
u_{j} \\
u_{k}
\end{array}\right]+\frac{l^{(e)}}{6}\left[\begin{array}{ll}
2 & 1 \\
1 & 2
\end{array}\right]\left[\begin{array}{c}
u_{j}^{\bullet} \\
u_{k}^{\cdot}
\end{array}\right]-\Delta \frac{l^{(e)}}{2}\left[\begin{array}{l}
1 \\
1
\end{array}\right]=0 .
$$

In order to get the difference equation at the node $i$, we write the element equations for two consecutive elements $y_{i-1} \leq y \leq y_{i}$ and $y_{i} \leq y \leq y_{i+1}$, assembling the resulting two element equations, one obtains

$$
\frac{1}{l^{(e)}}\left[\begin{array}{ccc}
1 & -1 & 0 \\
-1 & 2 & -1 \\
0 & -1 & 1
\end{array}\right]\left[\begin{array}{c}
u_{i-1} \\
u_{i} \\
u_{i+1}
\end{array}\right]+\frac{H l^{(e)}}{6}\left[\begin{array}{ccc}
2 & 1 & 0 \\
1 & 4 & 1 \\
0 & 1 & 2
\end{array}\right]\left[\begin{array}{c}
u_{i-1} \\
u_{i} \\
u_{i+1}
\end{array}\right]+\frac{l^{(e)}}{6}\left[\begin{array}{ccc}
2 & 1 & 0 \\
1 & 4 & 1 \\
0 & 1 & 2
\end{array}\right]\left[\begin{array}{c}
u_{i-1}^{\bullet} \\
u_{i}^{\bullet} \\
u_{i+1}^{\bullet}
\end{array}\right]=\Delta \frac{l^{(e)}}{2}\left[\begin{array}{l}
1 \\
2 \\
1
\end{array}\right]
$$

Putting row corresponding to the node $i$ to zero in Eq.(3.2) we obtain the following difference schemes with $l^{(e)}=y_{k}-y_{j}$ is the length of the element $(e),\left(y_{j} \leq y \leq y_{k}\right)$

$$
\frac{6}{l^{(e)^{2}}}\left(-u_{i-1}+2 u_{i}-u_{i+1}\right)+H\left(u_{i-1}+4 u_{i}+u_{i+1}\right)+\left(u_{i-1}^{\bullet}+4 u_{i}^{\bullet}+u_{i+1}^{\bullet}\right)=6 \Delta
$$


Applying the trapezoidal rule to Eq.(3.3) the following system of equations in Crank-Nicholson method are obtained with $l^{(e)}=h$

$$
A_{1} u_{i-1}^{j+1}+A_{2} u_{i}^{j+1}+A_{1} u_{i+1}^{j+1}=A_{3} u_{i-1}^{j}+A_{4} u_{i}^{j}+A_{3} u_{i+1}^{j}+\Omega_{l i}^{j} .
$$

Applying a similar procedure to the Eqs (2.10) and (2.11), we get

$$
\begin{aligned}
& B_{1} \theta_{i-1}^{j+1}+B_{2} \theta_{i}^{j+1}+B_{1} \theta_{i+1}^{j+1}=B_{3} \theta_{i-1}^{j}+B_{4} \theta_{i}^{j}+B_{3} \theta_{i+1}^{j}+\Omega_{2 i}^{j}, \\
& D_{1} C_{i-1}^{j+1}+D_{2} C_{i}^{j+1}+D_{1} C_{i+1}^{j+1}=D_{3} C_{i-1}^{j}+D_{4} C_{i}^{j}+D_{3} C_{i+1}^{j}
\end{aligned}
$$

where

$$
\begin{aligned}
& A_{1}=1-3 r+\frac{1}{2} r H h^{2} ; \quad A_{2}=4+6 r+2 r H h^{2} ; \quad A_{3}=1+3 r-\frac{1}{2} r H h^{2} ; \quad A_{4}=4-6 r-2 r H h^{2}, \\
& B_{1}=P_{r}-3 r+\frac{1}{2} r R h^{2} ; B_{2}=4 P_{r}+6 r+2 r R h^{2} ; B_{3}=P_{r}+3 r-\frac{1}{2} r R h^{2} ; B_{4}=4 P_{r}-6 r-2 r R h^{2}, \\
& D_{1}=\mathrm{Sc}-3 r+\frac{1}{2} r \delta \mathrm{Sc} h^{2} ; \quad D_{2}=4 \mathrm{Sc}+6 r+2 r \delta \mathrm{Sc} h^{2}, \\
& D_{3}=\mathrm{Sc}+3 r-\frac{1}{2} r \delta \mathrm{Sc} h^{2} ; \quad D_{4}=4 \mathrm{Sc}-6 r-2 r \delta \mathrm{Sc} h^{2}, \\
& \Omega_{l i}^{j}=6 k\left(\operatorname{Gr} \theta_{i}^{j}+G_{m} C_{i}^{j}\right) ; \quad \Omega_{2 i}^{j}=6 r \operatorname{Pr} \operatorname{Ec}\left(u_{i}^{j+1}-u_{i}^{j}\right)^{2} .
\end{aligned}
$$

Here, $r=k / h^{2}$ and $h, k$ are mesh sizes along the $y$-direction and time $t$-direction, respectively. Index $i$ refers to the space and $j$ refers to the time. In Eqs (3.4)-(3.6) taking $i=1(1) n$ and using boundary conditions (2.12), the following tri-diagonal systems of equations are obtained

$$
\begin{aligned}
& A u=A^{\prime}, \\
& B \theta=B^{\prime}, \\
& D C=D^{\prime}
\end{aligned}
$$

where $A, B$ and $D$ are tri-diagonal matrices of order - $n$ and whose elements are given by

$$
\begin{aligned}
& A_{i, i}=A_{2} ; \quad B_{i, i}=B_{2} ; \quad D_{i, i}=D_{2} \quad \text { at } \quad i=1(1) n, \\
& A_{i-1, i}=A_{i, i-1}=A_{1} ; \quad B_{i-1, i}=B_{i, i-1}=B_{1} ; \quad D_{i-1, i}=D_{i, i-1}=D_{1} \quad \text { at } \quad i=2(1) n .
\end{aligned}
$$

Here $u, \theta, C$ and $A^{\prime}, B^{\prime}, D^{\prime}$ are column matrices having $n$-components, namely $u_{i}^{j+1}, \theta_{i}^{j+1}, C_{i}^{j+1}$ and $u_{i}^{j}, \theta_{i}^{j}, C_{i}^{j}$, respectively. The above tri-diagonal systems of Eqs (3.7)-(3.9) are solved by using Thomas 
algorithm. The boundary condition $y \rightarrow \infty$ is approximated by $y_{\max }=10$, which is sufficiently large for the velocity to approach convergence criterion. The numerical solutions for the velocity, temperature and concentration profiles are obtained by using $C$ - program. To judge the convergence and stability of the Ritz finite element method, the same program was run with slightly changed values of $h$ and $k$, no significant change was observed in the values of velocity $(u)$, temperature $(\theta)$ and concentration $(C)$. Hence, we conclude that the Ritz finite element method is convergent and stable. The non-dimensional skin-friction at the plate is given by $\tau=\left(\frac{\partial u}{\partial y}\right)_{y=0}$.

\section{Results and discussion}

We have formulated and solved the problem of an unsteady MHD free convection with a parabolic starting motion of an infinite isothermal vertical porous plate. To gain a perspective of the physics of the flow regime, the velocity profiles, the temperature profiles and the concentration profiles as well as the skinfriction coefficient have been discussed by assigning various values to the physical parameters encountered in the problem under investigation. The value of $\operatorname{Pr}$ is taken to be 0.71 , which corresponds to air and the value of $\mathrm{Sc}$ is chosen to be 0.22 , which represents hydrogen at $25^{\circ} \mathrm{C}$ and one atmosphere pressure. During the numerical computations of the results, the values of the other physical parameters are considered as $\mathrm{Gr}=5.0, \mathrm{Gm}=5.0, \lambda=1.0, \delta=0.5, \mathrm{Ec}=0.1, \quad M=0.5, K=1.0$ and $t=0.2$.

Velocity profiles: Figure 1 illustrates the effects of the radiation parameter $\lambda(=1,2,3)$ on the velocity profiles. It is seen that the fluid velocity decreases with increasing values of the radiation parameter. The velocity of the fluid increases quickly near the plate and then decreases gradually to zero as $y \rightarrow \infty$. Figure 2 demonstrates the effects of the Eckert number $\operatorname{Ec}(=0.1,0.3,0.5)$ on the velocity profiles. The Eckert number defines the relationship between the kinetic energy in the flow and enthalpy. It embodies the conversion of kinetic energy into internal energy by the work done against the viscous fluid stress. The positive values of Eckert number indicate cooling of the plate, i.e., loss of the heat to the fluid from the plate. It is clear that an increase in the Eckert number causes a rise in the fluid velocity. Figure 3 shows the effects of the Schmidt number $\mathrm{Sc}$ on the velocity profiles for $\mathrm{Sc}=0.22$ (hydrogen), $\mathrm{Sc}=0.60$ (water-vapour), and $\mathrm{Sc}=0.78$ (ammonia). It is observed that an increase in the Schmidt number leads to a decrease in the velocity of the fluid, due to the decrease in the molecular diffusivity, which results in a decrease in the concentration and velocity boundary layer thickness. Figure 4 presents the effects of the chemical reaction parameter $\delta(=0.5,1.0,1.5)$ on the velocity profiles. It can be seen that the fluid velocity decreases with increasing the chemical reaction parameter. Figure 5 illustrates the effects of the magnetic parameter $M=(0.5,1.0,1.5)$ on the velocity profiles. The velocity curves show that the rate of transport is remarkably reduced with increasing values of the magnetic parameter. This result agrees qualitatively with the expectations, since the magnetic field exerts a retarding effect on free convection flows. The variation of the velocity profiles with the dimensionless permeability parameter $K(=1,2,3)$ is presented in Fig.6. This figure clearly indicates that the fluid velocity increases with increasing values of the permeability parameter. Physically, this result can be achieved when the holes of the porous medium are very large so that the resistance of the medium may be neglected. Figure 7 demonstrates the effect of the thermal Grashof number $\operatorname{Gr}(=5,7,9)$ on the velocity profiles. Here, the positive values of the thermal Grashof number correspond to cooling of the plate. It is observed that an increase in the value of the thermal Grashof number leads to an increase in the fluid velocity. This is due to the fact that buoyancy force enhances the fluid velocity and increases the boundary layer thickness with an increase in the value of the thermal Grashof number. Figure 8 illustrates the effects of the mass Grashof number $\mathrm{Gm}(=5,7,9)$ on the velocity profiles. As seen from this figure, the effects of $\mathrm{Gm}$ on the fluid velocity are same as that of Gr this fact is confirmed by comparing Figs 7 and 8 . Figure 9 is 
plotted to show the effects of the time parameter $t(=0.2,0.4,0.6)$ on the velocity profiles. It is observed that an increase in the value of time parameter $t$ increase the fluid velocity.

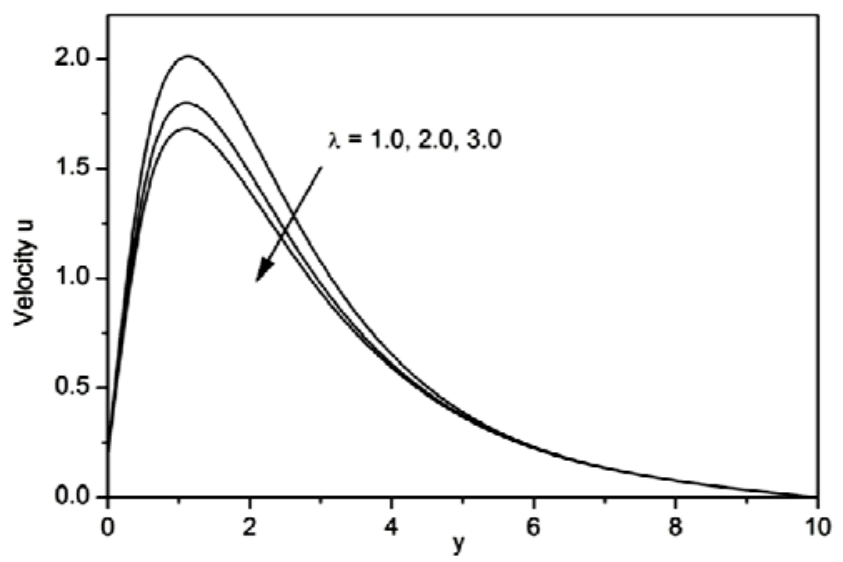

Fig.1. Effect of radiation parameter $(\lambda)$ on the velocity profiles.

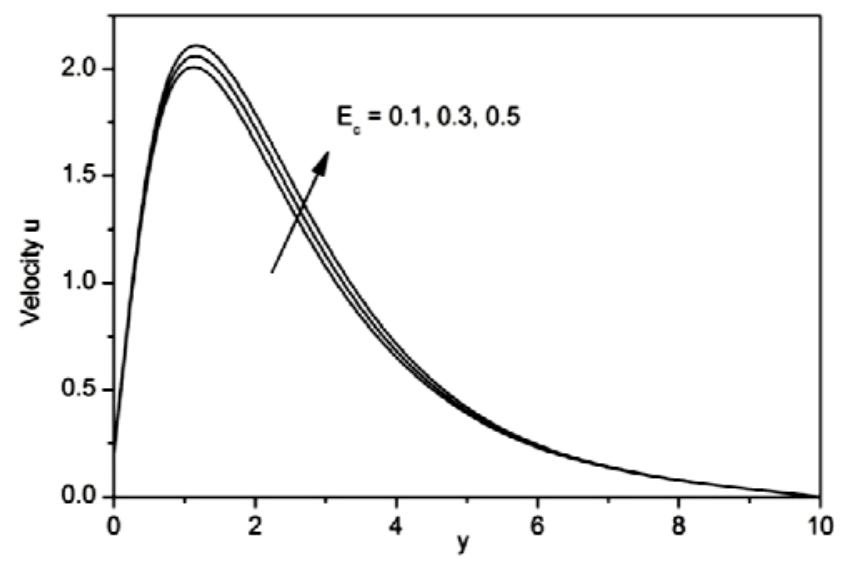

Fig.2. Effect of Eckert number (Ec) on the velocity profiles.

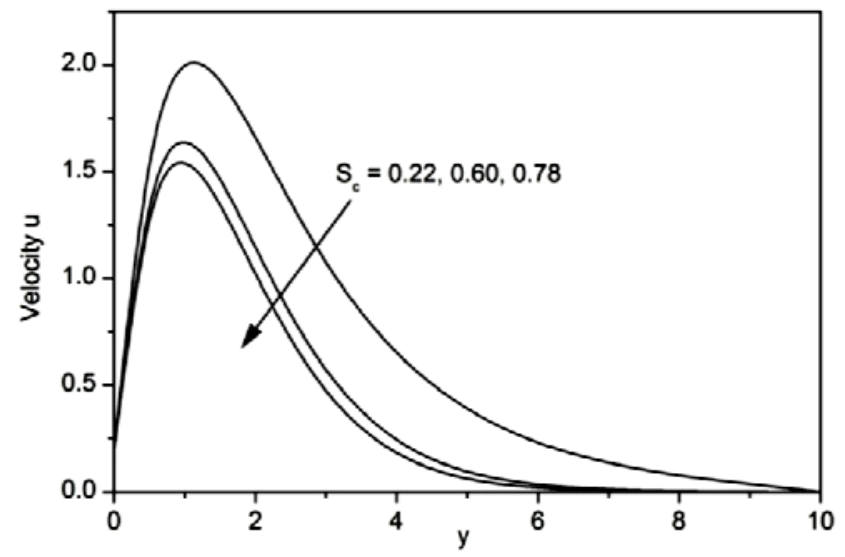

Fig.3. Effect of Schmidt number (Sc) on the velocity profiles. 


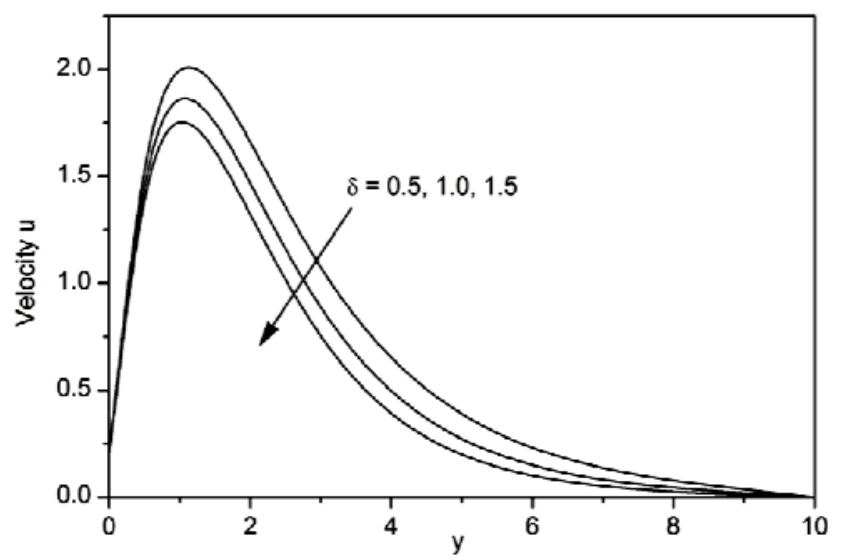

Fig.4. Effect of chemical reaction parameter $(\delta)$ on the velocity profiles.

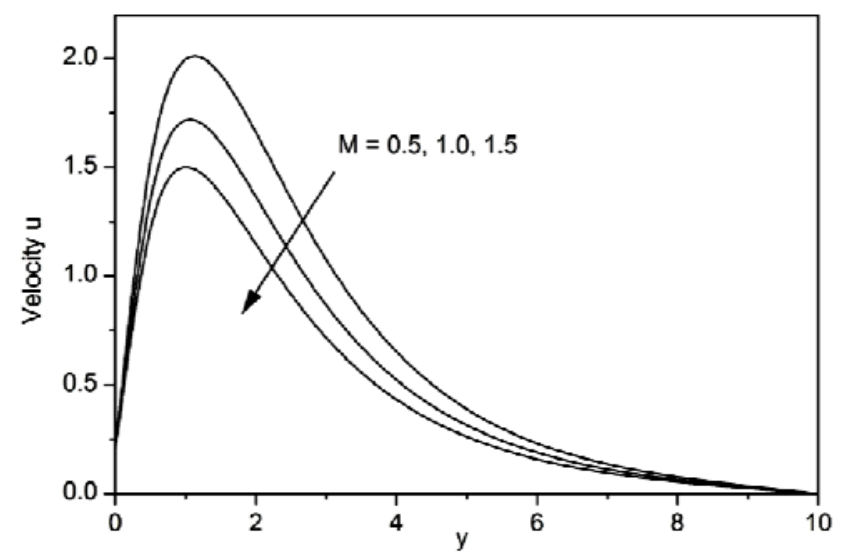

Fig.5. Effect of magnetic parameter $(M)$ on the velocity profiles.

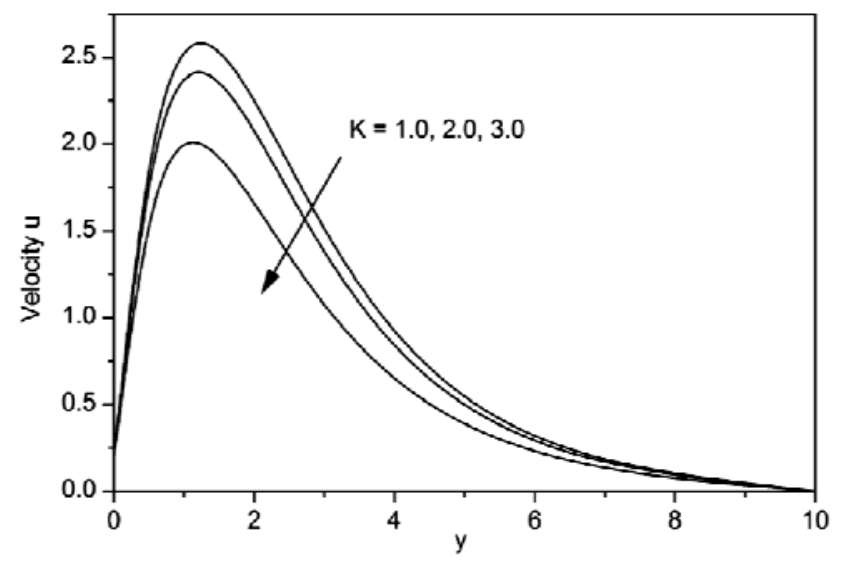

Fig.6. Effect of permeability parameter $(K)$ on the velocity profiles. 


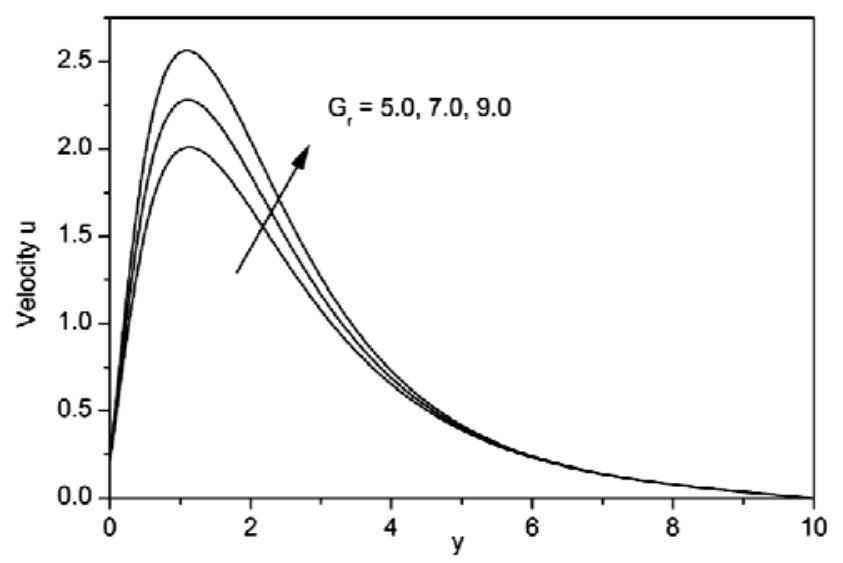

Fig.7. Effect of thermal Grashof number (Gr) on the velocity profiles.

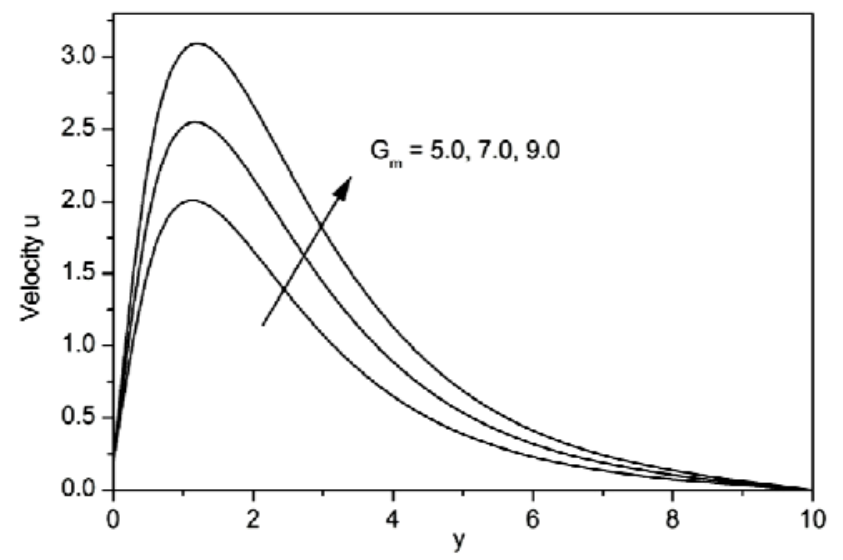

Fig.8. Effect of mass Grashof number $(\mathrm{Gm})$ on the velocity profiles.

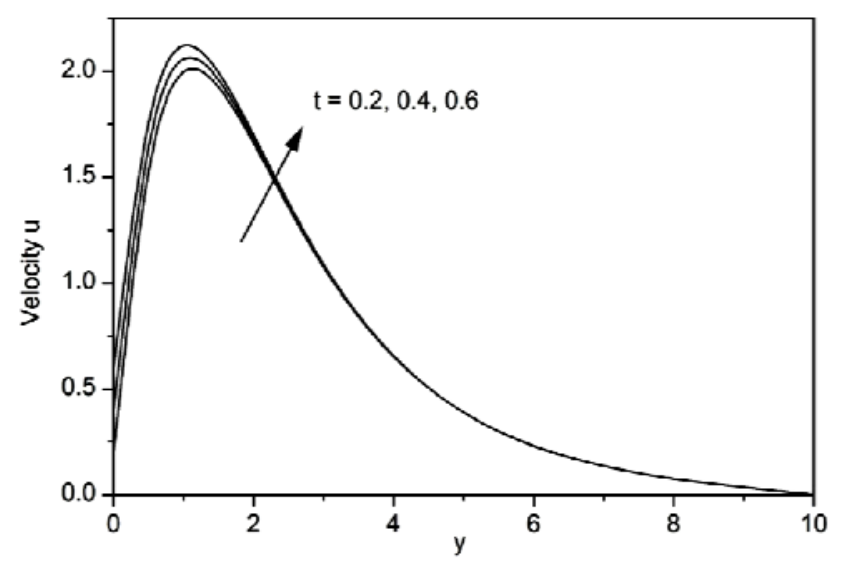

Fig.9. Effect of time parameter $(t)$ on the velocity profiles. 
Temperature profiles: Figure 10 represents the effects of the radiation parameter $\lambda(=1,2,3)$ on the temperature profiles. It is seen from this figure that there is a fall in the temperature profiles as the radiation parameter increased. The effects of the Eckert number $\operatorname{Ec}(=0.1,0.3,0.5)$ on the temperature profiles are presented in Fig.11. It is observed that an increase in the Eckert number leads to an increase in the temperature profiles.

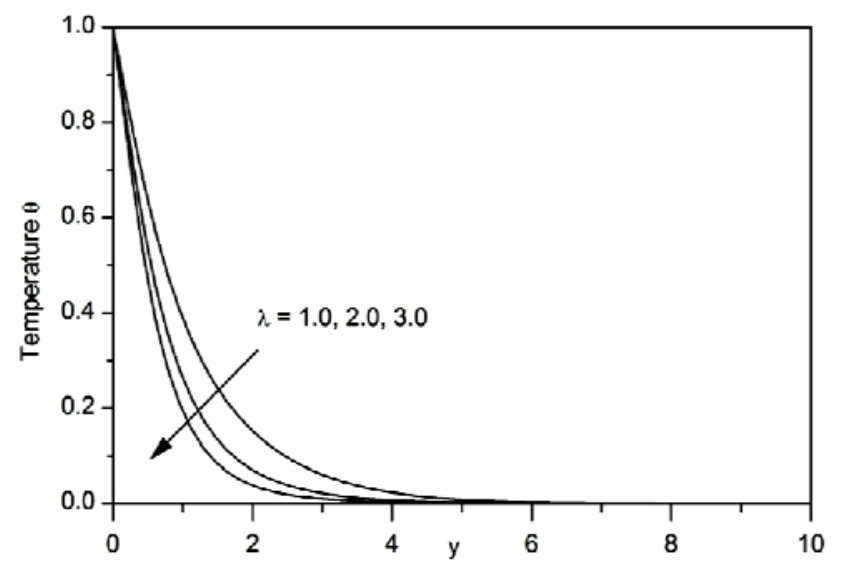

Fig.10. Effect of radiation parameter $(\lambda)$ on the temperature profiles.

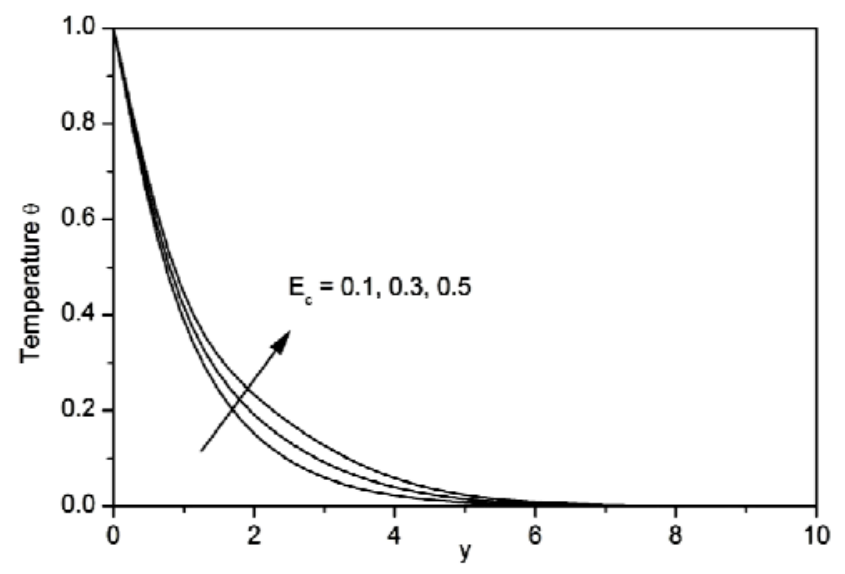

Fig.11. Effect of Eckert number (Ec) on the temperature profiles.

Concentration profiles: Figure 12 is plotted to show the effects of the Schmidt number Sc on the concentration profiles for different realistic values of $\mathrm{Sc}=0.22,0.60$ and 0.78 that are physically corresponds to hydrogen, water-vapour and ammonia, respectively. It is observed that the concentration decreases with increasing Schmidt number. Physically, the increase of the Schmidt number results in a decrease of molecular diffusion. Hence, the concentrations of the species is higher for small values of Sc and lower for large values of Sc. The influence of the chemical reaction parameter $\delta(=0.5,1.0,1.5)$ on the concentration profiles is shown in Fig.13. It is noticed from this figure that there is a marked effect of increasing values of $\delta$ on the concentration distribution in the boundary layer. It is clear that the increasing values of $\delta$ decrease the concentration of species in the boundary layer. Physically, large values of $\delta$ reduce the solutal boundary layer thickness and increase the mass transfer. 


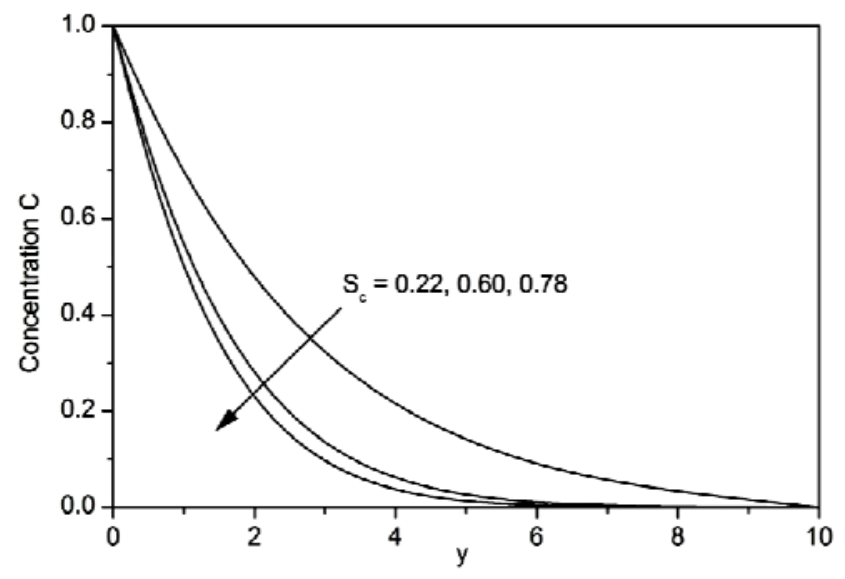

Fig.12. Effect of Schmidt number (Sc) on the concentration profiles.

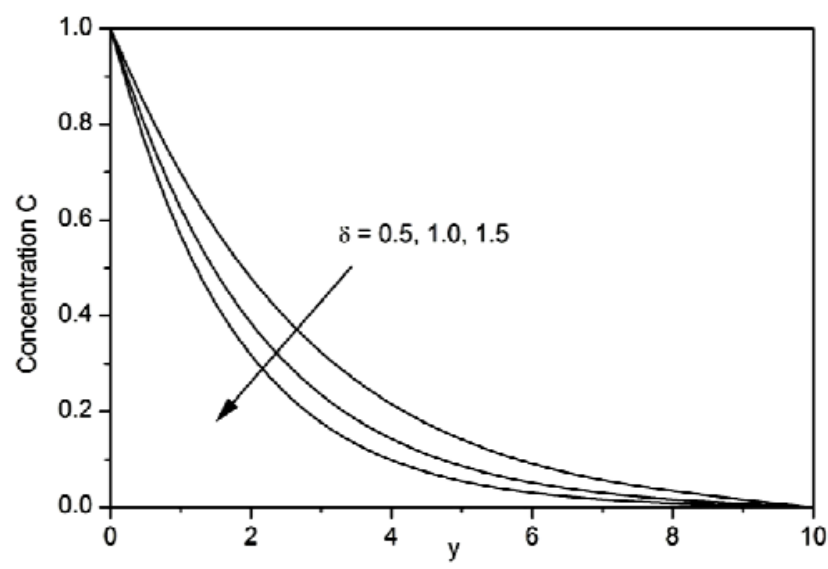

Fig.13. Effect of chemical reaction parameter $(\delta)$ on the concentration profiles.

Table 1 illustrates the effects of the radiation parameter $(\lambda)$, Schmidt number $(\mathrm{Sc})$ and Eckert number $(E c)$ on the skin-friction. From this table, it is observed that an increase in the value of the radiation parameter or Schmidt number decreases the skin-friction whereas an increase in the value of the Eckert number increases the skin-friction. The effects of the magnetic parameter $(M)$, permeability parameter $(K)$ and chemical reaction parameter $(\delta)$ on the skin-friction are presented in Tab.2. From this table, it is seen that the skin-friction decreases with increasing values of the magnetic parameter or chemical reaction parameter and increases with increasing values of the permeability parameter. Table 3 demonstrates the effects of the thermal Grashof number (Gr) mass Grashof number $(\mathrm{Gm})$ and time parameter $(t)$ on the skinfriction. From this table, it is clear that an increase in the thermal Grashof number or mass Grashof number or time parameter leads to an increase in the skin-friction. 
Table 1. Effects of $\lambda, \operatorname{Sc}$ and Ec on the skin-friction coefficient $(\tau)$.

\begin{tabular}{|c|cccc|}
\hline$\lambda$ & $\mathrm{Sc} / \mathrm{Ec}$ & 0.22 & 0.60 & 0.78 \\
\hline \multirow{4}{*}{1.0} & 0.1 & 3.039136 & 2.623584 & 2.503094 \\
& 0.3 & 3.095006 & 2.673220 & 2.550294 \\
& 0.5 & 3.153666 & 2.724524 & 2.598760 \\
& & & & \\
\multirow{4}{*}{3.0} & 0.1 & 2.739832 & 2.325818 & 2.206034 \\
& 0.3 & 2.771484 & 2.354778 & 2.234122 \\
& 0.5 & 2.804290 & 2.384346 & 2.262628 \\
& & & & \\
& 0.1 & 2.555062 & 2.141444 & 2.021912 \\
& 0.3 & 2.575442 & 2.160122 & 2.040286 \\
& 0.5 & 2.596398 & 2.179088 & 2.058850 \\
\hline
\end{tabular}

Table 2. Effects of $M, K$ and $\delta$ on the skin-friction coefficient $(\tau)$.

\begin{tabular}{|l|cccc|}
\hline$M$ & $K / \delta$ & 1.0 & 2.0 & 3.0 \\
\hline \multirow{4}{*}{0.5} & 0.5 & 3.039136 & 3.530054 & 3.727810 \\
& 1.0 & 2.880472 & 3.343024 & 3.529258 \\
& 1.5 & 2.751562 & 3.190728 & 3.367440 \\
& & & & \\
1.0 & 0.5 & 2.662750 & 3.039136 & 3.080194 \\
& 1.0 & 2.525522 & 2.880472 & 3.020642 \\
& 1.5 & 2.414212 & 2.751562 & 2.884692 \\
& & & & \\
& 0.5 & 2.366164 & 2.662750 & 2.777938 \\
& 1.0 & 2.245542 & 2.525522 & 2.634184 \\
& 1.5 & 2.147820 & 2.414212 & 2.517526 \\
\hline
\end{tabular}

Table 3. Effects of Gr, Gm and $t$ on the skin-friction coefficient $(\tau)$.

\begin{tabular}{|l|cccc|}
\hline $\mathrm{Gr}$ & $\mathrm{Gm} / t$ & 5.0 & 7.0 & 9.0 \\
\hline \multirow{5}{*}{5.0} & 0.2 & 3.039136 & 3.853738 & 4.672808 \\
& 0.4 & 3.252516 & 4.066144 & 4.884236 \\
& 0.6 & 3.466770 & 4.279420 & 5.096538 \\
& & & & \\
7.0 & 0.2 & 3.543440 & 4.367046 & 5.197042 \\
& 0.4 & 3.757002 & 4.579434 & 5.408060 \\
& 0.6 & 3.972180 & 4.793040 & 5.620290 \\
& & & & \\
& 0.2 & 4.057942 & 4.892190 & 5.734866 \\
& 0.4 & 4.272324 & 5.104800 & 5.945704 \\
& 0.6 & 4.488260 & 4.318962 & 6.258092 \\
\hline
\end{tabular}




\section{Conclusion}

In this work, the problem of unsteady MHD free convection with a parabolic starting motion of an infinite isothermal vertical porous plate in the presence of radiation and chemical reaction is discussed. The governing equations of the problem are solved numerically by applying the Ritz finite element method. We conclude that:

- the velocity of the fluid increases with increasing the Eckert number or permeability parameter or thermal Grashof number or mass Grashof number or time parameter;

- an increase in the radiation parameter or Schmidt number or chemical reaction parameter or magnetic parameter decreases the fluid velocity;

- the fluid temperature decreases with increasing the radiation parameter and increases with increasing the Eckert number;

- the fluid concentration decreases with increasing the values of the Schmidt number and chemical reaction parameter;

- the skin-friction increases with an increase in the Eckert number or permeability parameter or thermal Grashof number or mass Grashof number or time parameter;

- the skin-friction decreases with an increase in the radiation parameter or Schmidt number or magnetic parameter or chemical reaction parameter.

\section{Nomenclature}

C - dimensionless concentration

$C^{\prime}-$ species concentration in the fluid

$C_{w}^{\prime}$ - species concentration at the plate

$C_{\infty}^{\prime}-$ concentration in the fluid far away from the plate

$c_{p}$ - specific heat at constant pressure

$D$ - mass diffusion

$\mathrm{Gm}$ - mass Grashof number

$\mathrm{Gr}$ - thermal Grashof number

$g-$ acceleration due to gravity

$K$ - porosity parameter

$k$ - thermal conductivity

$M$ - magnetic parameter

Pr -Prandtl number

Sc - Schmidt number

$u$ - dimensionless velocity

$T^{\prime} \quad$ - temperature of the fluid

$T_{\infty}^{\prime} \quad$ - temperature of the fluid far away from the plate

$T_{w}^{\prime} \quad$ - temperature at the plate

$t^{\prime}$ - time

$u^{\prime}-$ velocity components in the $x^{\prime}$ direction

$\beta-$ volumetric coefficient of thermal expansion

$\beta^{*}-$ volumetric coefficient of concentration expansion

$\delta$ - chemical reaction parameter

$\theta$ - dimensionless temperature

$v-$ kinematic viscosity

$\rho$ - fluid density

$\sigma$ - electrical conductivity 


\section{Subscripts}

$w \quad$ - conditions at the wall

$\infty \quad$ - free stream conditions

\section{References}

[1] Muthucumaraswamy R. and Janakiraman B. (2006): MHD and radiation effects on moving isothermal vertical plate with variable mass diffusion. -Theoret. Appl. Mech., vol.33, pp.17-29.

[2] Shanker R.T. and Gnaneshwar R.M. (2007): Radiation effects on MHD flow past an impulsively started infinite vertical plate through a porous medium with variable temperature and mass diffusion. - J. Pure and Appl. Phys., vol.19, No.3, pp.191-200.

[3] Bear J. (1998): Dynamics of Fluids in Porous Media. - New York: Dover.

[4] Kaviany M. (1993): Principles of Heat Transfer in Porous Media. - New York: MacGraw-Hill.

[5] Ahmed N. and Sarmah H.K. (2009): Thermal radiation effects on a transient MHD flow with mass transfer past an impulsively fixed infinite vertical plate. -Int. J. Appl. Math and Mech., vol.5, No.5, pp.87-98.

[6] Mahmoud M.A.A. (2009): Thermal radiation effects on unsteady MHD free convection flow past a vertical plate with temperature dependent viscosity. -Canadian J. Chem. Engg, vol.87, pp.47-52.

[7] Mukhopadhyay S. (2009): The effects of thermal radiation on unsteady mixed convection flow and heat transfer over a porous stretching surface in porous medium. - Int. J. Heat and Mass Transfer, vol.52, pp.3261-3265.

[8] Sharma S. and Deka R.K. (2012): Thermal radiation and oscillating plate temperature effects on MHD unsteady flow past a semi-infinite porous vertical plate under and chemical reaction. - Int. J. Phys. and Math. Sci, vol.2, No.2, pp.33-52.

[9] Muralidharan M. and Muthucumaraswamy R. (2013): Radiation effects on linearly accelerated plate with variable mass diffusion in the presence of magnetic field. - Applied Mathematical Science, vol.7, No.113, pp.5645-5656.

[10] Muthucumaraswamy R. and Ganesan P. (2001): Effect of the chemical reaction and injection on flow characteristics in an unsteady upward motion of an isothermal plate. - J. Appl. Mech. Tech. Phys, vol.42, No.4, pp.665-671.

[11] Chamkha A.J. (2003): MHD flow of a uniformly stretched vertical permeable surface in the presence of heat generation/absorption and a chemical reaction. - Int. Commun. Heat and Mass Transfer, vol.30, No.3, pp.413422.

[12] Kandasamy R., Periasamy K. and Prabhu K.K. (2005): Effects of chemical reaction, heat and mass transfer along $a$ wedge with heat source and concentration in the presence of suction or injection. - Int. J. Heat and Mass Transfer, vol.48, No.7, pp.1388-1394.

[13] Ibrahim F.S, Elaiw A.M. and Bakr A.A. (2008): Effects of chemical reaction and radiation absorption on the unsteady MHD free convection flow past a semi-infinite vertical permeable moving plate with heat source and suction. - Commun. in Non-Linear Sci. Numerical Simulation, vol.13, No.6, pp.1056-1066.

[14] Muthucumaraswamy R. and Janakiraman B. (2008): Mass transfer effects on isothermal vertical oscillating plate in the presence of chemical reaction. - Int. J. Appl. Math and Mech., vol.4, No.1, pp.66-74.

[15] Mahapatra N., Dash G.C. Panda S. and Acharya M. (2010): Effects of chemical reaction on free convection flow through a porous medium bounded by a vertical surface. - J. Eng. Phys and Thermo-Phys, vol.83, No.1, pp.130140 .

[16] Mohamed R.A., Osman N.A.N. and Abo-Dahadb S.M. (2013): Unsteady MHD double-diffusive convection boundary layer flow past a radiative hot vertical surface in a porous media in the presence of chemical reaction and heat sink. - Meccanica, vol.48, pp.931-941. 
[17] Muthucumaraswamy R. and Sivakaumar P. (2016): MHD flow past a parabolic flow past an infinite isothermal vertical plate in the presence of thermal radiation and chemical reaction. - Int. J. Appl. Mech. and Engg, vol.21, No.1, pp.95-105.

[18] Reddy J.N. (2005): An introduction to the finite element method. $-3^{\text {rd }}$ Ed., New York: Mac-Graw Hill.

[19] Gebhart B. (1962): Effects of viscous dissipation in natural convection. - Journal of Fluid Mechanics, vol.14, pp. 225-232.

[20] Gebhart B. and Mollendorf J. (1969): Viscous dissipation in external natural flows. - Journal of Fluid Mechanics, vol.38, pp.79-107.

[21] Soundalgekar V.M. (1972): Viscous dissipation effects on unsteady free convective flow past an infinite vertical porous plate with constant suction. - Int. J. Heat and Mass Transfer, vol.15, pp.1253-1261.

[22] Gupta A. S, Pop I and Soundalgekar V. M. (1979): Free convection effects of the flow past an accelerated vertical plate in an incompressible dissipative fluid. - Rev. Rom. Sci. Techn-Mec. Apl., vol.24, pp.561-568.

[23] Takhar H.S., Beg O.A. and Kumari M. (1998): Computational analysis of coupled radiation-convection dissipation non-gray gas flow in a non-Darcy porous medium using the Keller-Box implicit difference scheme. Int. J. Energy Res, vol.22, pp.141-159.

[24] Zueco Jordan J. (2007): Network simulation method applied to radiation and viscous dissipation effects on MHD unsteady free convection over vertical porous plate. - Appl. Mathematical Modeling, vol.31, No.20, pp.20192033.

[25] Prabhakar Reddy B. (2016): Mass transfer effects on an unsteady MHD free convective flow of an incompressible viscous dissipative fluid past an infinite vertical porous plate. - Int. J. Appl. Mech. and Eng., vol.21, No.1, pp.143-153.

Received: January 26, 2018

Revised: December 3, 2018 